It has now been possible to confirm that about 30 per cent of one strand of SV40 DNA (that which is transcribed in vitro by $E$. coli polymerase) is transcribed during lytic infections before viral DNA replication occurs to yield stable "early" SV40 RNA; whereas, after viral DNA replication begins, in addition to these early RNA sequences about 60 per cent of the complementary DNA strand is transcribed into stable late SV40 RNAs. These findings imply that a strand-switching event occurs during the regulated transcription of the SV40 genome as the virus replicates in permissive cells. According to Patch et al. (Proc. US Nat. Acad. Sci., 69, 3375 ; 1972) the crucial segment of the SV40 genome that contains this transcriptional control region may be accessible to investigation in a non-defective human adenovirus 2-SV40 hybrid virus.

Patch, Lewis and Levine have been working with an Ad2-SV40 hybrid virus which is called $A d 2+N_{1}$. This virus has a genome which contains some 10-18 per cent of the SV40 genome (estimates of the amount of SV40 DNA vary with the techniques used to measure it) covalently linked to a human adenovirus 2 genome carrying a 5 per cent deletion. When this hybrid virus infects monkey cells and replicates some SV40 early RNA is transcribed and SV40-specific $U$ antigen is induced as it is early during infections of monkey cells with intact SV40. These observations indicate that at least some of the SV40 DNA that is transcribed early during replication is present in the segment of the SV40 genome in the Ad2 $+N_{1}$ hybrid. That this is indeed the case has been confirmed by Patch et al. who first devised a method for separating the two strands of $\mathrm{Ad} 2+$ ND, DNA and then showed that SV40 specific RNA transcribed in vitro by $E$. coli RNA polymerase hybridizes to one of the two strands of hybrid virus DNA.

But are any of the late SV40 DNA sequences also present in the small segment of SV40 DNA in the $\mathrm{Ad} 2+$ ND 1 genome? To answer this question Patch et al. challenged the separated strands of hybrid virus DNA with SV40-specific RNA extracted at late times from cells infected with SV40 and supporting its replication. This RNA contains both early and late sequences and some of it hybridizes to one strand of $\mathrm{Ad} 2+\mathrm{ND}_{1}$ DNA while some of it hybridizes to the other $\mathrm{Ad}^{+} \mathrm{ND}_{1}$ DNA strand. In other words, the SV40 DNA in the hybrid virus contains sequences complementary to both early and late SV40 RNAs. This must mean that the 10-18 per cent of the SV40 genome in the hybrid virus must include the SV40 early/late transcriptional control region. Clearly this hybrid virus and the four others belonging to the same family are proving to be more than virologist's curiosities; they are tools for probing further the nature of the signals which initiate and/or terminate transcription of SV40 DNA.

Another useful tool for analyses of the structure and function of SV40 DNA is described in a crop of reports in the same issue of the Proceedings (ibid., 3215, 3365, 3370, 3448). All these reports are concerned with one property or another of the $R_{1}$ restriction endonuclease that can be isolated from $E$. coli carrying the drug resistance transfer factor $\mathrm{RTF}_{1}$. This enzyme converts circular duplex SV40 DNA into linear unit length duplex molecules by cleaving both DNA strands at a unique site which can be mapped with respect to other physical markers and by partial denaturation mapping. Obviously the last has not been heard of this enzyme or the non-defective adenovirus 2-SV40 hybrid viruses which could, for example, be teamed together in some interesting experiments.

\section{CONTRACEPTION Switching off the Male}

by our Special Correspondent "THE only world for global survival is the contraceptive world." Male contraception should be culturally acceptable and completely effective; it should be non-toxic, have specific and reversible action and have no effects upon male libido and complete fulfilment of sexual union. These were the conclusions of a workshop on "Contraception: The Masculine Gender", organized by Schering AG in Berlin, from November 29 to December 2. The organizers had gathered together a diverse group of scientists with a wide range of interests in an attempt to answer the question "Why bother with the male?" The fact of the matter is that contraception, with the advent of the oral steroid pills, has become the responsibility of the woman; the more difficult problem of male contraception has been largely ignored.

The testis and epididymis are the chief target sites for contraceptive action. Maleness begins with the differentiation of the testes early in foetal life and must impose over femaleness. Dr A. Jost (University of Paris) discussed this development and stressed the importance of the Sertoli cells, which appear quite suddenly in the testis, swell and within a few hours completely encompass the germ cells. The control of this process is not yet known but, together with the interstitial cells, the Sertoli cells probably "fight" the female problem.

An unusual feature of the Sertoli cell membrane was presented by Drs D. Fawcett and B. Gilula (Harvard Medical School). In order for any agent to affect spermatogenesis it must penetrate the seminiferous tubules. The epithelium, which at some point must allow upward movement of new spermatocytes, has two compartments-a basal region to which materials have direct access and an adluminal region with developing cells to which substances are denied access by the Sertoli cell junctions. These junctional complexes are unlike any other epithelium and at some places the apposing cell membranes are very close and appear to fuse to form a blood/testis barrier. Beautiful freezeetched pictures of these areas were presented from experimental rodents. This technique cleaves the cell membrane to reveal the outwardly directed inner mem. brane ( $A$ face) and the inwardly directed outer membrane ( $B$ face). Cleavage at

\title{
Locating Yeast Ribosomal RNA Cistrons
}

IN next Wednesday's Nature New Biology (December 27), Finkelstein, Blamir and Marmur report the development of a technique for analysing intact chromosomal DNA molecules in yeast. After labelling, intact DNA molecules are released on the surface of sucrose gradients by detergent lysis of spheroplasts. By these means it is estimated that the linear duplex molecular weight of yeast DNAs lies between 400 and $700 \times 10^{6}$.

It is known that the size of yeast rDNA is at the upper limit of this molecular weight range and that in most organisms rRNA cistrons are clustered so that it is thus possible that all the rDNA is located on one chromosome.

DNA-rRNA hybridization experiments showed, however, that it is more probable that all the rDNA is accommodated on two chromosomes, one of which contains mostly rDNA. A likely candidate for the latter chromosome is yeast chromosome 1 because it contains only one known genetic marker. Comparison of labelled DNA in a monosomic $(2 n-1)$ strain of yeast, haploid only for chromosome 1 , with DNA of its isogenic diploid showed that the molecular weight of this chromosome is of the right magnitude. Control hybridization experiments showed that none of the other species of DNA in this size class hybridized to rRNA. Ribosomal DNA is thus located on chromosome 1 .

Other experiments showed that the rDNA content of chromosome 1 accounts for 70 per cent of the total rRNA genes and there is evidence that the remaining 30 per cent of the rRNA genes are also clustered on one or more chromosomes. 